\title{
Al Acceleration of HEP Collider Simulation
}

\section{Tara Leininger (Lafayette College/FNAL), Kevin Pedro (FNAL), Lena Franklin (UMD), Yiheng Ye (U Chicago)}

\section{Motivation}

- Detector simulation is a critical part of High Energy Physics (HEP), but is computationally expensive

- Generative Neural Networks (GANs) have been explored as a machine learning solution (Ref. [1])

- GANs can generate new simulated events from a large database of existing events, but could have mathematical and practical concerns

- We use of a Convolutional Neural Network (CNN) as a solution

- This CNN takes in quickly generated low-quality events and enhances them to a more useful resolution (See Fig. 1)

\section{Introduction}

- A CNN is an artificial intelligence architecture used to classify images (ex. Search engines)

- Inspired by Ref. [2] (See Fig. 2) we:

- Feed the CNN a low-quality image

- Train it to predict the true energy for each pixel

- Have the CNN to return a higher quality image (regression, rather than classification)

- We train out CNN so we can simulate high quality data quickly by generating low quality events and enhancing them using the trained network

\section{Model and Training}

- Our CNN has 9 convolutional layers and 3x3 kernels with $\mathrm{N}=100$ features

- A simplified approach has worked in the past, where a CNN was used to remove noise artificially added to high quality simulated events

- This approach was successful Ref. [3]

- In this project, we build out that framework to enhance actual low-quality events with simplifications

- We use single photon events generated at $850 \mathrm{MeV}$

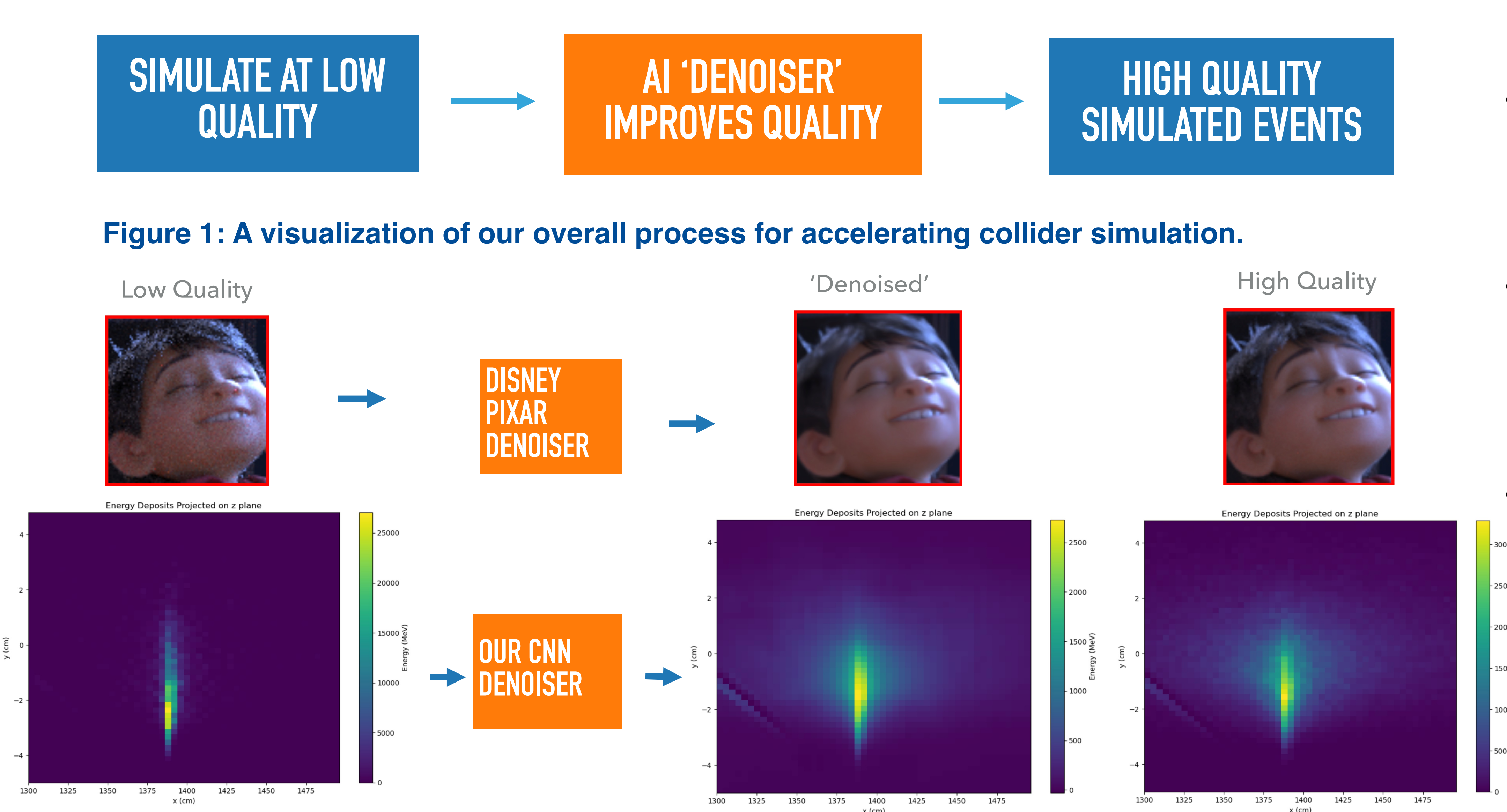

Figure 2: This use of a CNN was inspired by a similar regression algorithm used by Disney enhancing images for films, we enhance photon simulation data

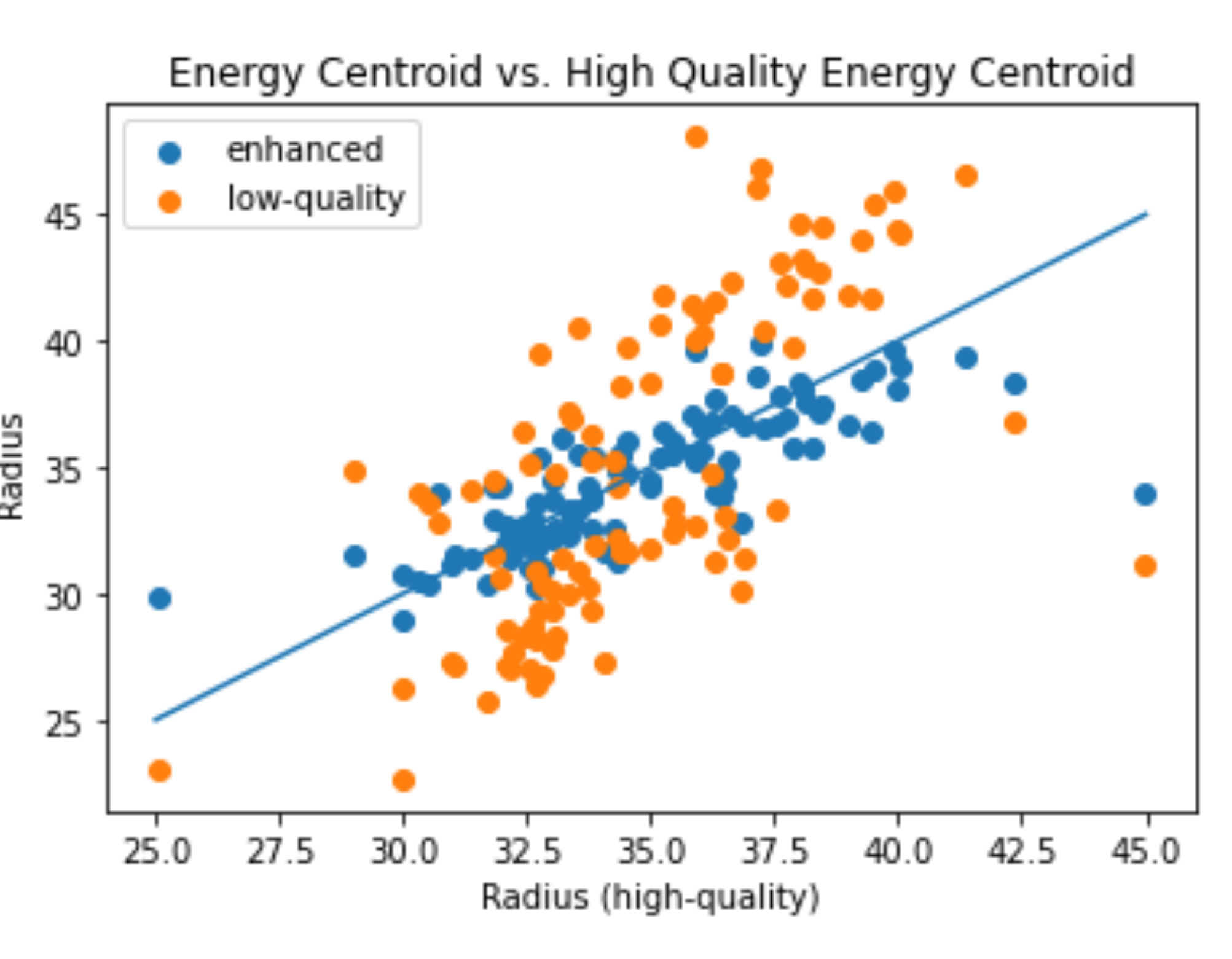

Figure 3: Compares the energy centroid of both the enhanced and low-quality data against the high-quality data. The line,
perfect correspondence

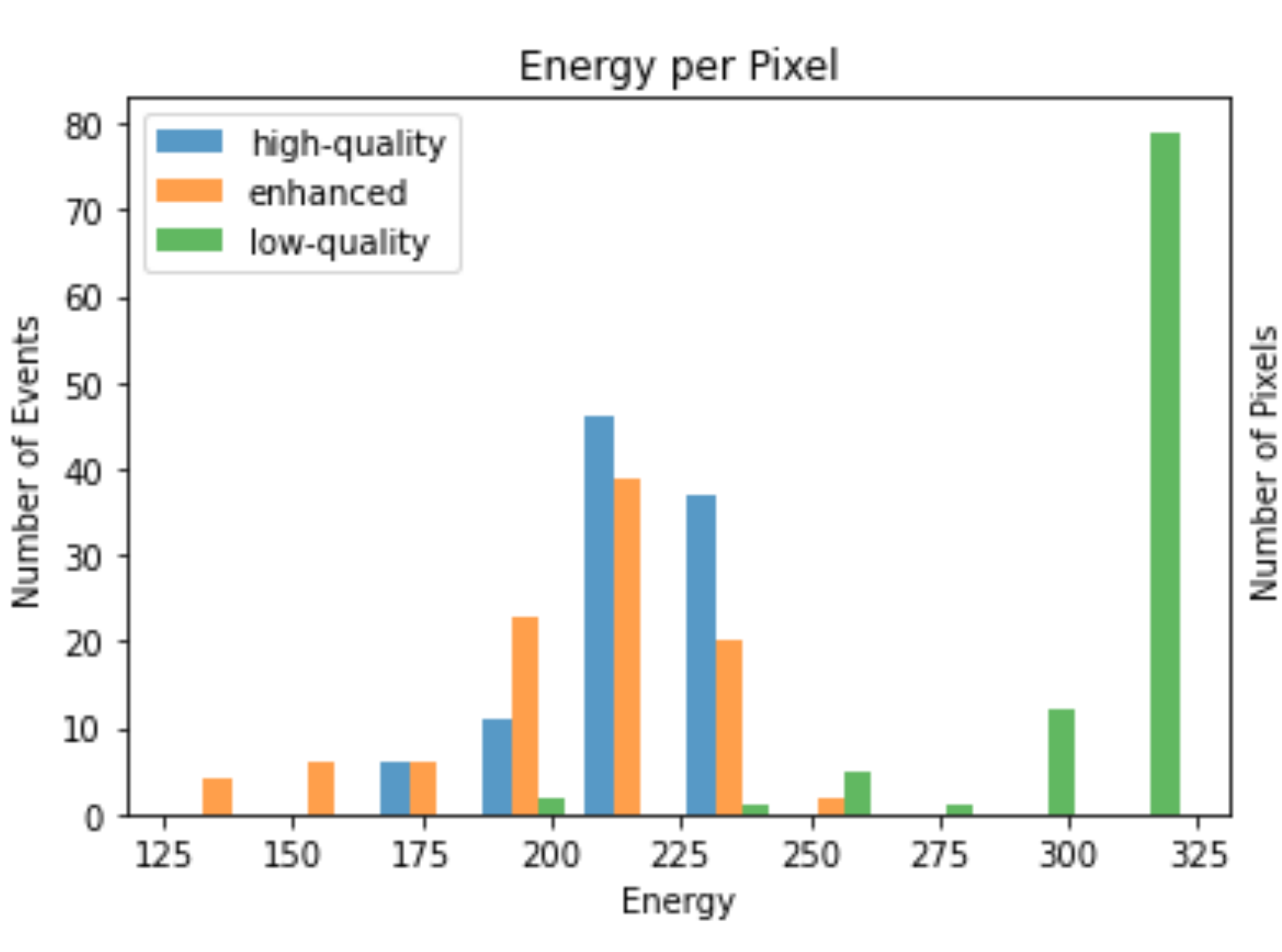

Figure 5: Average energy in an event. Again, enhanced and high-quality match much mo
closely than with low-quality.

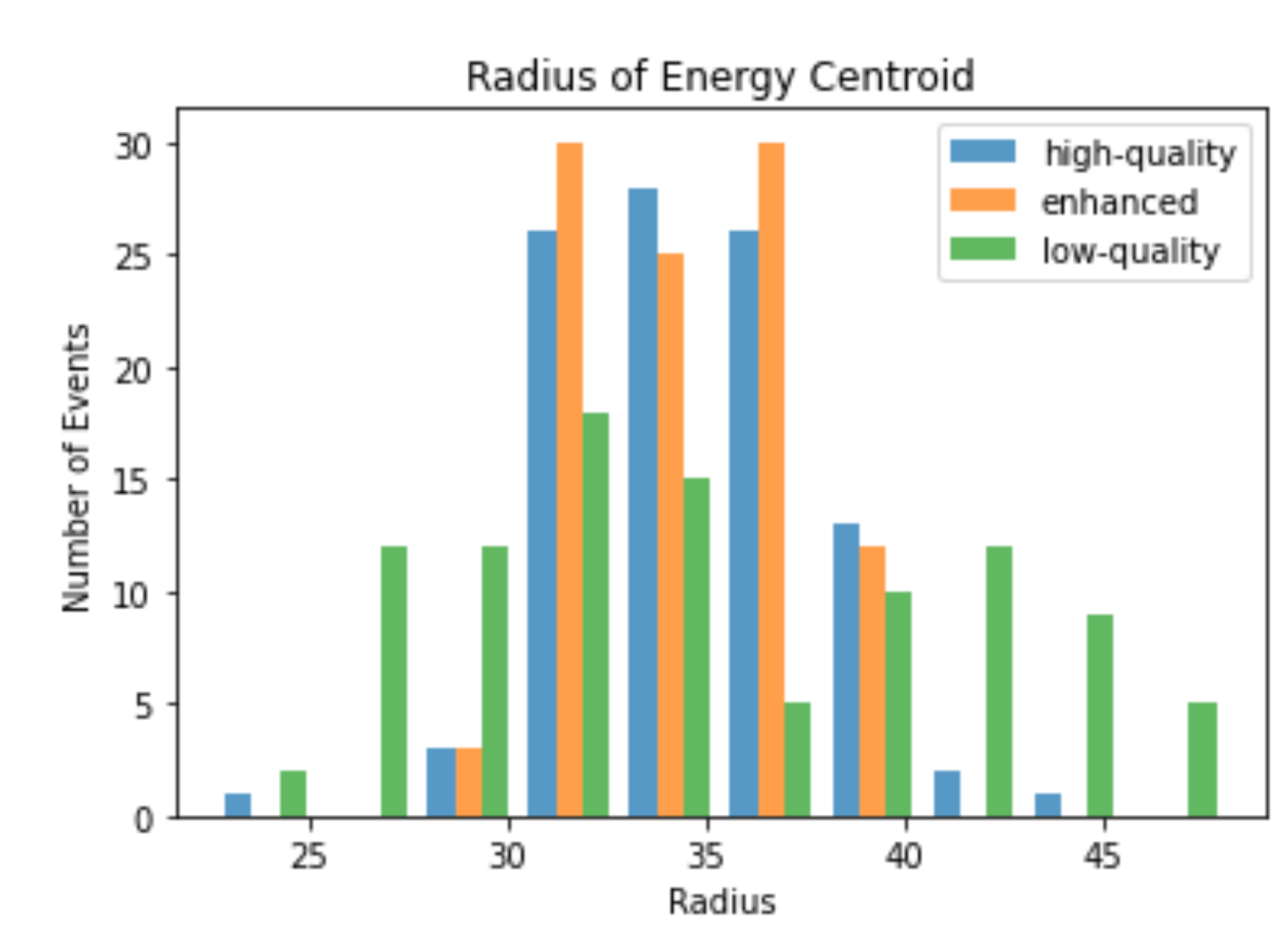

Figure 4: Energy centroid for high-quality, lowquality, and CNN enhanced event. Enhanced than the low-quality

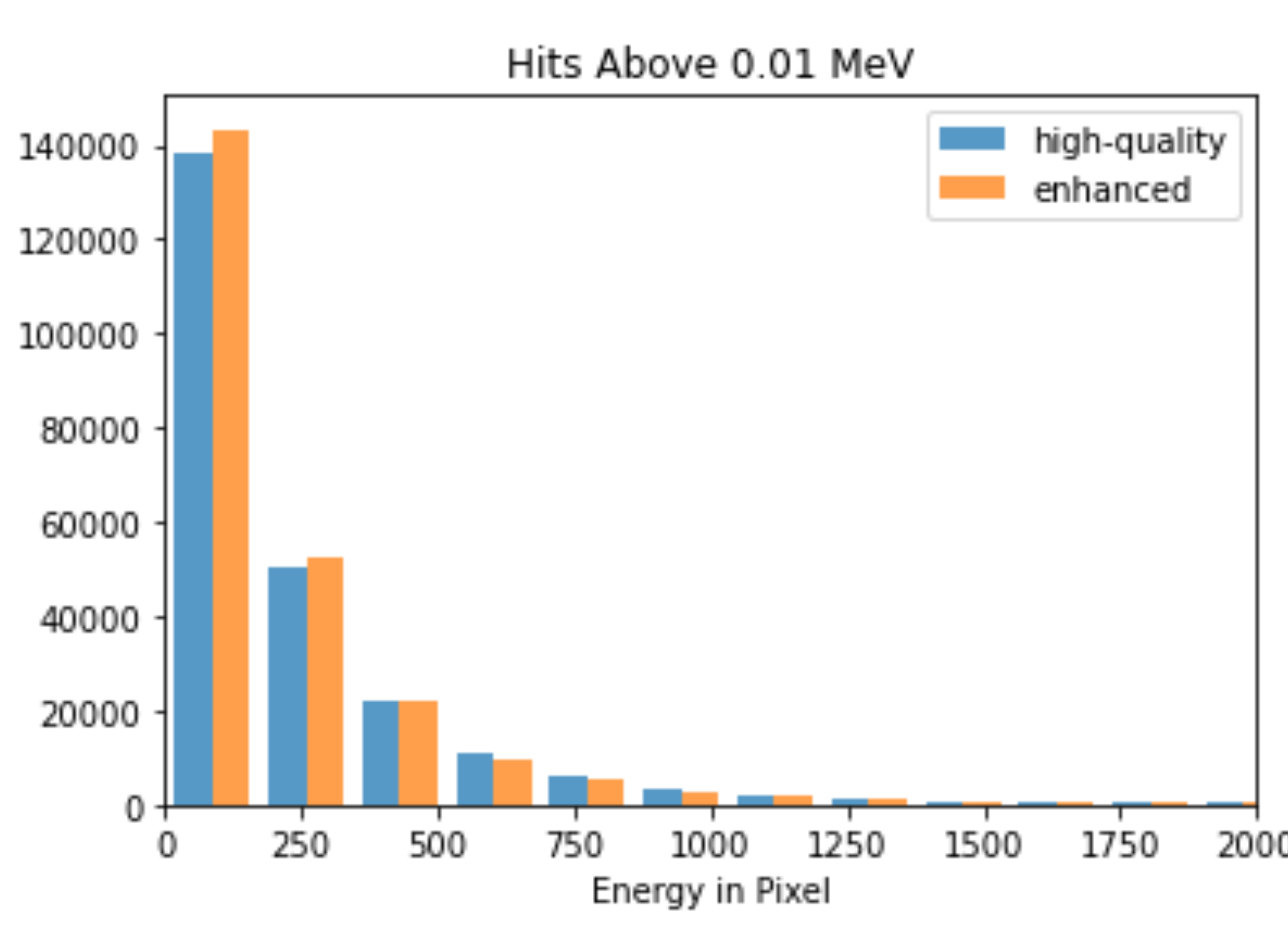

Figure 6: Distribution of energies of individual pixels, given energies are above $0.01 \mathrm{MeV}$. High
match closely.

\section{Analysis}

- We want to generate a reasonable physical prediction, not predict a particular image, so pixel-by-pixel comparison may not be the only useful metric

- Previous metrics for Al simulation Ref. [1]

- centroid of the energy data

- number of bins above a given energy threshold

We use these same metrics, but as we use a CNN and not a GAN, we can compare high quality, low quality, and CNN-enhanced events metrics against each other Enhanced images more closely match energy centroid data (see Fig. 3 \& 4) than low-quality input data

Also match aggregate energy data well (Fig. 5 \& 6)

\section{Future Work}

- In the future, working with more types of desired simulated events (as opposed to just single-photon) would make this approach more usable

- The structure of the approach could be modified to incorporate an image classifier which would sort between real high quality simulated events, and lowquality events enhanced by our CNN

- Satisfying this image classifier would mean we had been successful in training our CNN

We plan to add more input features (in addition to energy) work with 3D data

- Examine different neural network architectures

ACKNOWLEDGEMENTS REFERENCES

I would like to thank my

research mentor Kevin Pedro

for his help and guidance. 1

would also like to thank the

following for contributing to

studies for low quality Geant 4

events: Sunanda Banerjee

(FNAL), Scarlet Norberg,

Angel Rosado Trinidad, Brian

Cruz, Harold Guerrero, Sudhi

Malik (University of Puerto

Rico Mayaguez)
1. E. Buhmann, S. Diefenbacher, E. Eren, F. Kruger Comput. Softw. Big Sci.5, no.1, 13 (2021) [arXiv:2005.05334 [physics.ins-det]] 2. Bako, S., Vogels, T., Mcwilliams, B., Meyer, M., Novák, J., Harvill, A., Sen, P., Derose, T., \& Rousselle, F. (2017). Kernel-Predicting Convolutional Networks for Denoising Monte Carlo Rend

3. Presenter Franklin, Lena $(2020,7) A$ Presenter, Franklin, Lena. (2020, 7)
Denoising to Accelerate Detector Simulation. [Poster Presentation]. Fermilab, Simulation. . Poster
Batavia, Ilinois. 